\title{
Erratum to: Tolerability of High-Volume Subcutaneous Injections of a Viscous Placebo Buffer: A Randomized, Crossover Study in Healthy Subjects
}

\author{
Clapton Dias, ${ }^{1,4}$ Bassam Abosaleem, ${ }^{1}$ Caroline Crispino, ${ }^{1}$ Bing Gao, ${ }^{1,2}$ and Adam Shaywitz ${ }^{1,3}$
}

Author affiliations were inadvertently switched in the published article. The correct author affiliations are:

(1) Amgen Inc., 1 Amgen Center Drive, Thousand Oaks, California 91320, USA

(2) Currently at Gilead Sciences, Foster City, California, USA

(3) Currently at BioMarin Pharmaceutical, Inc., San Rafael, California, USA

The online version of the original article can be found at http:// dx.doi.org/10.1208/s12249-015-0288-y.

${ }^{1}$ Amgen Inc., 1 Amgen Center Drive, Thousand Oaks, CA 91320, USA.

${ }^{2}$ Gilead Sciences, Foster City, CA, USA.

${ }^{3}$ BioMarin Pharmaceutical, Inc., San Rafael, CA, USA.

${ }^{4}$ To whom correspondence should be addressed. (e-mail: claptondias@gmail.com) 